\title{
EVALUATION OF CHANGES IN THE PARAMETERS OF BODY STABILITY IN THE PARTICIPANTS OF A NINE-DAY SNOWBOARDING COURSE
}

\author{
MICHAŁ STANISZEWSKI', PRZEMYSŁAW ZYBKO', IDA WISZOMIRSKA² \\ Józef Pitsudski University of Physical Education in Warsaw, Faculty of Physical Education, \\ Department of Water and Winter Sports', Faculty of Rehabilitation, Department of Anatomy and Kinesiology 2
}

Mailing address: Michał Staniszewski, Józef Piłsudski University of Physical Education, Department of Water and Winter Sports, 34 Marymoncka Street, 00-968 Warsaw, tel.: +48 228340431 ext. 227, fax: +48 22 8651080, e-mail: michal.staniszewski@awf.edu.pl

\begin{abstract}
Introduction. Snowboarding is a sports discipline in which postural control is key to achieving an effective technique. The body is positioned sideways on the snowboard, with only the head facing forward. This study evaluated the effect of several days of intense snowboarding on the parameters of static and dynamic body stability in persons with different levels of skill. Material and methods. A nine-day snowboarding course was designed and conducted with beginner $(\mathrm{N}=16)$ and advanced snowboarders $(\mathrm{N}=14)$ in the mountains in winter. Before and after the course, dynamic body stability was measured on a Biodex Balance System (USA) platform with an unstable surface, and static body stability was measured on a FreeMed Sensor Medica (Italy) stabilometric platform. Results. Measurements on an unstable surface showed significantly weaker ( $<<0.01$ ) values of body stability in a lateral stance in the snowboarding stance than in a forward stance and a significant $(\mathrm{p}<0.05)$ improvement in performance after the course. On a stable surface, the improvement in performance $(p<0.05)$ occurred only among the beginners, in the snowboard basic position. Conclusions. The results confirm that snowboarding, or continuous unstable balance, improves postural control, which leads to the conclusion that the lateral stance on the snowboard is a clearly disruptive factor in natural postural control. This constitutes a considerable difficulty, especially for beginners, who in addition to learning new technical skills, must adapt to continuously shifting balance.
\end{abstract}

Key words: body posture control, balance, biofeedback, postural stability, stable surface, unstable surface

\section{Introduction}

Control over vertical posture requires information collected from the surroundings by the receptors of sight, touch, pressure, and the vestibule of the ear, and by proprioceptive receptors as well. The information is sent to the central nervous system, where it is processed. Subsequently, nerve signals travel to the eyes and the muscles of the trunk and limbs, initiating reflexes that coordinate body posture $[1,2]$. De Oliveira et al. [3] interpret balance control as the combined effect of the central nervous system, senses (touch, sight, and balance), biomechanical limitations, cognitive processes, perception of verticality, and movement strategy. Research conducted by Meyer et al. [4] indicates that plantar feedback becomes one of the primary moderators of the correct body posture whenever the receptors of sight are inactive. Plantar feedback allows for a continuous adjustment of the position of the body in relation to the surroundings [5]. Other important factors in balance control are the elasticity of the soft tissues and the mechanics of the foot. A correct stable posture is required for locomotion and most voluntary movements.

Both static and dynamic postural stability can be tested [6-8]. However, as there are no practical means to assess COM displacements, postural stability tests use indirect indicators. Static balance tests are conducted on measurement devices with a stable, rigid surface, with COM displacements given by the oscillations of the centre of pressure (COP) of the feet. Subject literature assumes that COP oscillations correspond to the degree of balance. The most frequently analysed parameter is the length of the path designated by the movement of the COP of the feet [9-12]. Dynamic balance tests use measurement devices with an unstable surface. Balance is maintained through controlled adjustments to the position of the body and the assessment is based on the overall stability index (OSI) [13-16] .

One of the activities in which postural control plays a key role is snowboarding. Getting on a snowboard involves fastening both boots into the bindings, which immobilises the feet and excludes the lower limbs from the process of maintaining balance. The body is positioned sideways on the snowboard, with only the head facing forward and the eyes looking forward. The direction of the stance depends on the choice of the lead leg, or the leg that is in front during the ride. This stance is called the basic position. Some snowboarders prefer to ride with their right leg in front, that is with the right side of the body facing the slope and their head turned right, while others prefer to ride with their left leg in front. The choice is individual for each snowboarder. The opposite position, that is with the nonlead leg in front, is also possible. This is called the fakie position. However, this stance makes free riding difficult and should only be used by advanced snowboarders. The lateral position 
required for snowboarding is not natural for human locomotion. As a result of this positioning, the ride takes place within the human temporal plane, rather than, as with most natural and sports-related movements, within the sagittal plane [17, 18].

The immobilised feet make it difficult to maintain a vertical posture on the slope, and turning requires specific technical skills. In order to turn, a snowboarder presses down on one of the edges of the snowboard by shifting his or her body either toward the toes or toward the heels, that is in the sagittal plane. The lateral position means that snowboarders remain constantly in unstable balance, and turning involves a controlled loss of balance in the intended direction [19].

Many hours of snowboarding and training exercises improve control over the human-snowboard-snow system. It seems that, as training continues, body balance also improves, as indicated by the fact that snowboarders fall less often, are more secure on their boards, and are able to ride more effectively on increasingly demanding terrain. However, it is unknown whether the improved postural control during snowboarding is related to mechanisms involved with the improvement of body stability or with the improvement in general motor coordination involved in acquiring new technical skills. The subject literature provides no answer to this issue, and studies that analyse balance in winter sports are rare. This study aimed to investigate whether several days of intense snowboarding generates changes in static and dynamic body stability and whether snowboarding skills contribute to these changes. To this end, a nine-day snowboarding course was designed and conducted with beginner and advanced snowboarders in the mountains in winter.

\section{Material and methods}

All participants were informed about the research procedure, conditions of participation, dangers, and obligations. All participants gave written consent for participation in person, and they were informed that they would be able to opt out of the experiment at any point and with no consequences. The study was approved by the local Ethics Committee for Scientific Research.

\section{Participants}

Study participants comprised 30 university students of physical education who took part in a snowboarding course. The participants were non-randomly assigned to two groups according to their level of skill (Tab. 1). The group of beginner snowboarders (BEG) comprised 16 persons who declared that they had no snowboarding skills prior to the course. The group of advanced snowboarders (ADV) comprised 14 persons with several years of snowboarding experience, who, at the time, were participating in a snowboarding instructor course. All participants took part in a nine-day snowboarding camp. The beginner group learned to ride the snowboard using basic techniques, with the lead leg positioned always in front. The advanced group learnt advanced manoeuvres in both the basic position and the fakie

Table 1. Characteristics of the groups examined in the study

\begin{tabular}{|c|c|c|c|c|}
\hline Group & $\mathbf{N}$ & $\begin{array}{c}\text { Age } \\
\text { (years) }\end{array}$ & $\begin{array}{c}\text { Body mass } \\
(\mathbf{k g})\end{array}$ & $\begin{array}{c}\text { Height } \\
(\mathbf{c m})\end{array}$ \\
\hline BEG & $16(7$ men, 9 women) & $21.4 \pm 1.3$ & $68.1 \pm 13.5$ & $173.3 \pm 10.6$ \\
\hline ADV & $14(9$ men, 5 women) & $23.2 \pm 1.8$ & $71.5 \pm 11.1$ & $175.4 \pm 9.3$ \\
\hline
\end{tabular}

*statistically significant differences between groups $(\mathrm{p}=0.004)$. position. The two groups showed no significant differences in mean body mass or height. However, the advanced snowboarders were older than the beginners by an average of two years.

\section{Procedures}

In order to assess the effect of the course on postural control, static body stability and dynamic stability were measured before and after the course.

Dynamic body stability was measured on a Biodex Balance System SD (USA) device with an unstable surface. Three protocols were performed at Stability Level 8. Each protocol consisted of four 20-second trials with a 10 -second break in-between. The participants underwent a postural stability test (PST) in a twoleg forward stance with biofeedback with eyes open and closed, and in a lateral stance with the head facing the biofeedback screen, that is in positions that imitated both the basic position and the fakie position in snowboarding. For analysis of stability, the overall stability index (OSI) was used.

Static body balance was measured on a Sensor Medica FreeMed (Italy) stabilometric mat. Four 30-second protocols in a barefoot stance were performed: a two-leg stance with eyes open, a two-leg stance with eyes closed, a two-leg stance with eyes open and the head turned in the basic position, and a twoleg stance with the head turned in the fakie position. Analysis and comparisons were based on the path lengths of the COP of the feet.

\section{Statistical analysis}

The data obtained were subjected to statistical analysis using the Statistica Ver. 12 software package. Normality of distribution was assessed using the Shapiro-Wilk $w$ test. Changes in the parameters of stability were assessed using the repeated measures ANOVA. The probability level of $\mathrm{p}<0.05$ was assumed as statistically significant.

\section{Results}

During the test performed on an unstable surface with biofeedback, both groups showed the best stability in a forward stance with eyes open (Fig. 1 and 2). As expected, standing with eyes closed proved to be the most difficult test, yielding a more than three-fold decrease in the ability to maintain a stable posture. Interestingly, the lateral alignment of the body with only the head facing the screen significantly $(p<0.01)$ worsened postural control in both the basic and the fakie position and in both groups. On the other hand, neither group showed differences between the two directions in a lateral stance. Measurements conducted on the Body Balance System also revealed no statistically significant differences in the parameters of body stability between the two groups.

In the beginner group (Fig. 1), the snowboarding course improved postural control in a forward stance with eyes open $(\mathrm{p}<$ $0.05)$ and in a lateral stance with the head turned in the direction of the basic position ( $\mathrm{p}<0.05)$. In the advanced group (Fig. 2 ), a significant improvement was observed in a two-leg stance with eyes open $(\mathrm{p}<0.01)$ and in a lateral stance in the basic position $(\mathrm{p}<0.01)$ and the fakie position $(\mathrm{p}<0.05)$.

Measurements of postural stability conducted on a stable surface revealed significant differences in the path length of the COP in the beginner group (Fig. 3) between standing with eyes open and standing with eyes closed $(\mathrm{p}<0.01)$ as well as between the former and the snowboard basic position ( $\mathrm{p}<0.05)$. In the advanced group (Fig. 4), significant differences $(\mathrm{p}<0.05)$ occurred only between standing with eyes open and eyes closed. 


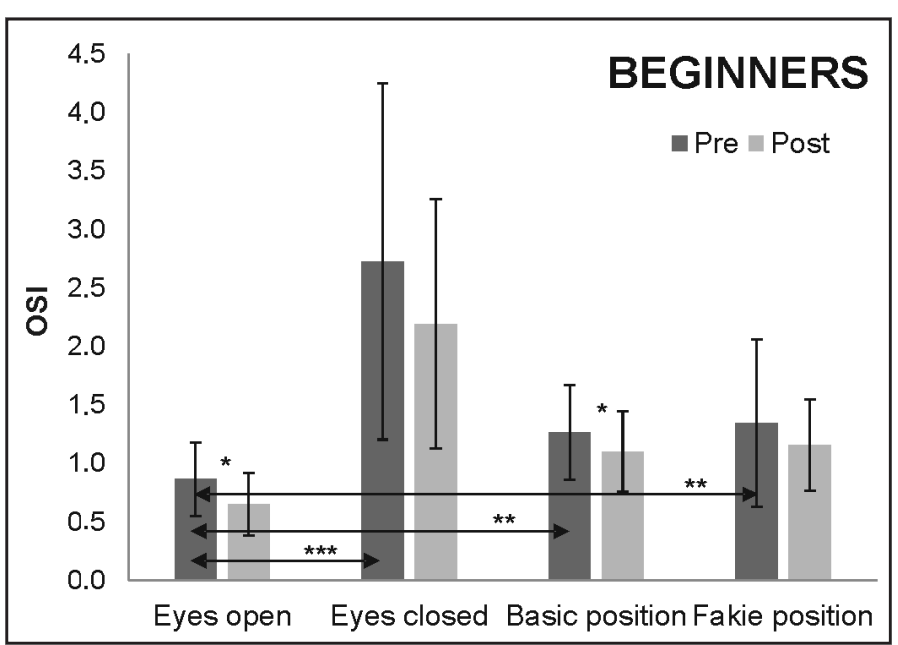

*statistically significant differences at the level of $\mathrm{p}<0.05$; ** statistically significant differences at the level of $\mathrm{p}<0.01$; ${ }^{* * *}$ statistically significant differences at the level of $\mathrm{p}<0.001$.

Figure 1. Overall stability index, OSI (mean values \pm SD), measured when standing on an unstable surface with biofeedback in the beginner group pre and post training camp

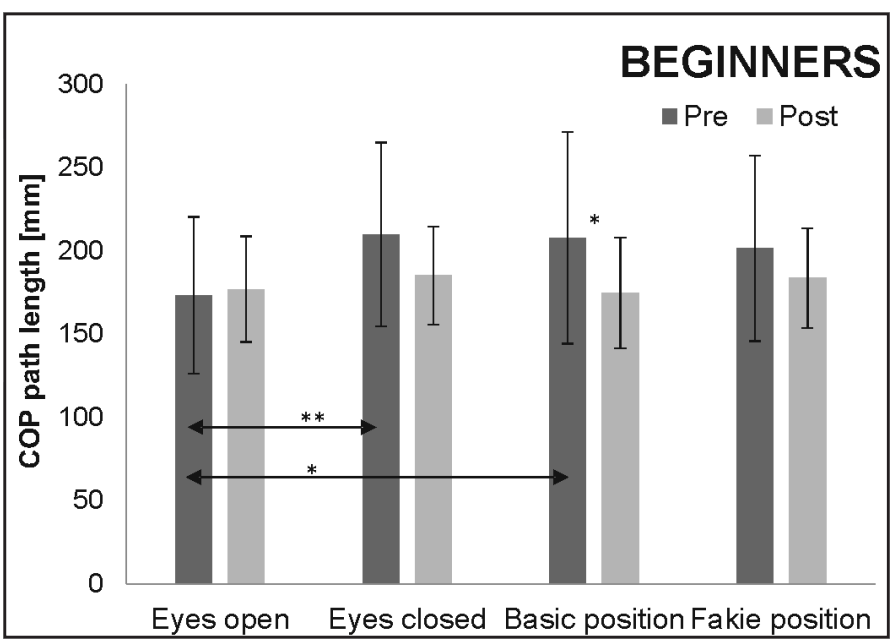

*statistically significant differences at the level of $\mathrm{p}<0.05$; ** statistically significant differences at the level of $\mathrm{p}<0.01$.

Figure 3. Path length of the COP of the feet (mean values \pm SD) measured while standing on a stable surface in the beginner group pre and post training camp

After the training camp, the beginner group showed changes in the path length of the COP only in a lateral stance in the basic position $(\mathrm{p}<0.05)$. In the advanced group there were no significant differences in postural stability measurements before and after the camp. No statistically significant differences were observed between the two groups, either.

\section{Discussion}

An assessment of static and dynamic body stability showed no statistically significant differences between the beginner

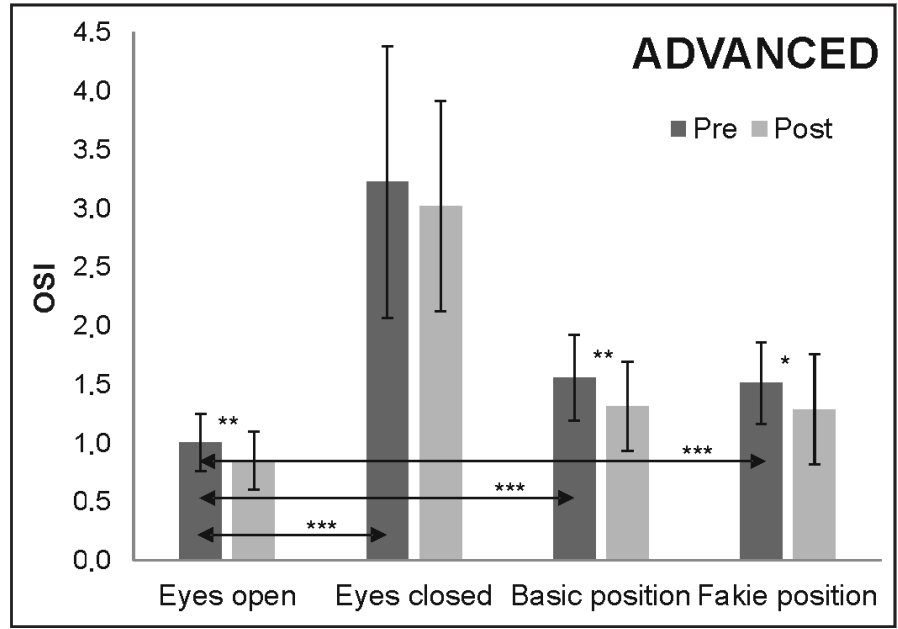

*statistically significant differences at the level of $\mathrm{p}<0.05$; ** statistically significant differences at the level of $\mathrm{p}<0.01 ;{ }^{* * *}$ statistically significant differences at the level of $\mathrm{p}<0.001$.

Figure 2. Overall stability index, OSI (mean values \pm SD), measured when standing on an unstable surface with biofeedback in the advanced group pre and post training camp

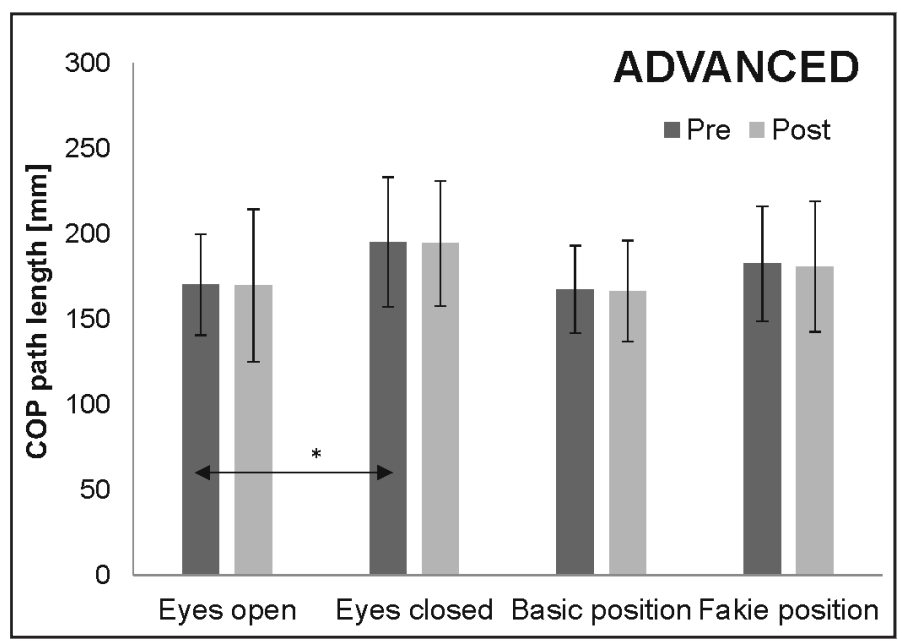

*statistically significant differences at the level of $\mathrm{p}<0.05$.

Figure 4. Path length of the COP of the feet (mean values \pm SD) measured while standing on a stable surface in the advanced group pre and post training camp

snowboarders and the advanced snowboarders. Similar findings were reported in studies conducted by other authors, who indicate that the level of skill does not always differentiate athletes on the basis of body balance. In many sports disciplines, such as shooting, soccer, or golf, the length of training experience has a positive effect on body balance. However, there are disciplines in which the level of technical skill does not differentiate athletes in terms of body stability. These disciplines include surfing, judo, alpine skiing, and, indeed, snowboarding [20, 21].

An interesting finding is that both beginner and advanced snowboarders performed worse in terms of stability in a lateral 
stance than in a forward stance. The only difference between the two stances is the turning of the head, which in the lateral stance faces a screen with biofeedback. Nonetheless, it is the turning of the head that causes a conflict between information about the vertical posture that originates in the head and arrives through the vestibular and sight systems and information that comes from the set of proprioceptors located in postural muscles. During a forward stance, all three of these mechanisms regulate balance within the same planes: A/P displacements are regulated within the sagittal plane, and $\mathrm{M} / \mathrm{L}$ displacements are regulated within the temporal plane. On the other hand, in the snowboarding stance in which the head is turned by 90 degrees toward the slope, displacements of the body toward the toes or the heels constitute displacements in the sagittal plane. However, from the perspective of the vestibular and sight systems, which regulate the orientation of the head relative to the surroundings, these displacements constitute displacements in the temporal plane. Such a conflict occurring when a snowboarder assumes the snowboarding stance and moves sideways, rather than forward, makes learning and riding difficult not only in snowboarding, but in other sports that require riding or surfing sideways on a board. Anthony et al. [22] pointed out a similar phenomenon in their study, in which they assessed surfers using a Biodex Balance System device with an unstable surface. As with this study, the balance tests were conducted in a lateral position relative to simulating the surfing stance, with only the head turned toward the screen with biofeedback. They demonstrated that the lateral position causes significant differences in body stability between the surfers depending on which leg they choose as their lead leg when standing on a surfboard.

In tests conducted on a stable surface, that is under conditions that differ to a greater extent from those experienced during snowboarding, differences between a forward stance and a lateral stance occurred only in the beginner group. This may indicate that the lack of such differences in the advanced group resulted from their considerable technical skill and the related adaptation to controlling their balance during snowboarding. Čech [23], who studied balance in ice hockey players, reached a similar conclusion. He conducted an experiment in which he observed no differences in postural stability, as measured on a stable surface, between a group of players who underwent special balance training and a group who underwent traditional training. Long-term hockey training was found to allow the players to adapt to difficult conditions of postural control during ice skating, and additional balancing exercises had no effect on the players' postural stability in static conditions. In contrast, when additional balancing exercises were applied throughout 12 weeks of training in field hockey, a discipline in which the surface stimulates body posture less strongly than in ice skating, they improved the players' static as well as dynamic body stability [24].

The results of the tests conducted before and after the nineweek snowboarding course indicate that improvement in the snowboarding technique strictly correlates with improvement in postural control. Of course, this is not the only factor that affects performance in snowboarding. Nonetheless, the statistically significant differences in the parameters of body stability before and after the course suggest that body balance plays a major role in snowboarding. To put it differently, snowboarding, or constant exposure to unstable balance, has a significant, positive effect on body stability.

Tests conducted in a standing position on a platform with an unstable surface showed significant changes in both the beginner and the advanced groups. The beginners improved their stability in a forward stance with eyes open and in a lateral stance in the basic position. In other words, in addition to general body balance, the improvement also concerned the lateral position, which the participants assumed when snowboarding. The advanced snowboarders additionally showed an improvement in the fakie stance, that is in the position with the opposite leg in front, as riding in this manner is a usual part of advanced training.

During the nine-day snowboarding course, the participants, especially the beginners, performed difficult exercises that involved maintaining a stable position on the snowboard. These were balancing exercises that helped to improve the participants' postural control. Studies conducted by other authors confirm that even a short-term period of balancing exercises may benefit body stability. Karakaya et al. [25] observed significant changes in postural stability following two weeks of proprioceptive training with persons not engaged in sports. Vando et al. [26] applied one week of coordination training in 10-yearold karate fighters, noting significant changes in body stability, as measured on a stable platform. In turn, Matin et al. [27] reported an improvement in both static and dynamic stability in children aged 10-12 years following four weeks of sensorimotor training.

\section{Conclusions}

The research conducted indicates that the lateral snowboarding stance relative to the direction of riding is a clearly disruptive factor to natural postural control mechanisms. This constitutes a considerable difficulty, especially for beginners, who in addition to learning new technical skills, must adapt to a continuously shifting balance. Furthermore, remaining in unstable balance can be considered a benefit to postural control. It is worth noting that, along with an improvement in general stability, improvement was also observed in areas that were trained during the nine-day snowboarding course, that is in the basic position for the beginners and the basic and reverse (fakie) positions for the advanced snowboarders.

Furthermore, it was found that analysis of changes in the parameters of body stability in advanced snowboarders should involve measurements on an unstable surface, as they reflect natural snowboarding activity better. As Williams et al. [28] state, because tests performed in static conditions are a suboptimal choice for advanced athletes who have already achieved good balancing skills in their sports careers, tests should be performed on an unstable surface instead. Zemková [29] notes that static balance is significant in sports such as shooting or archery. In contrast, in sports that involve moving on a board, such as snowboarding, skateboarding, or windsurfing, dynamic postural control is a key part of the movement technique and should be tested in dynamic conditions. Williams et al. [30] claim that compared to athletes who train on a stable surface, athletes who train on an unstable surface use a more effective strategy of maintaining stable posture in response to disruptions in balance.

\section{Acknowledgements}

This study was financed by the Polish Ministry of Science and Higher Education under research project DS. 199 of Józef Piłsudski University of Physical Education in Warsaw. 


\section{Literature}

1. Guskiewicz K.M., Perrin D.H. (1996). Research and clinical applications of assessing balance. Journal of Sport Rehabilitation 5(1), 45-63.

2. Wiszomirska I., Kaczmarczyk K., Błażkiewicz M., Wit A. (2015). The impact of a vestibular-stimulating exercise regime on postural stability in people with visual impairment. Biomed Research International Article ID: 136969. DOI: $10.1155 / 2015 / 136969$.

3. De Oliveira C.B., De Medeiros Í.T., Ferreira N.A., Greters M.E., Conforto A.B. (2008). Balance control in hemiparetic stroke patients: Main tools for evaluation. Journal of Rehabilitation Research E Development 45(8), 1215-1226.

4. Meyer P.F., Oddsson L.E., De Luca C.J. (2004). The role of plantar cutaneous sensation in unperturbed stance. Experimental Brain Research 156(4), 505-512.

5. Kavounoudias A., Roll R., Roll J.P. (1998). The plantar sole is a 'dynamometric map' for human balance control. Neuroreport 9(14), 3247-3252.

6. DiStefano L.J., Clark M.A., Padua D.A. (2009). Evidence supporting balance training in healthy individuals: A systemic review. Journal of Strength and Conditioning Research 23(9), 2718-2731.

7. Gribble P.A., Hertel J., Plisky P. (2012). Using the Star Excursion Balance Test to assess dynamic postural-control deficits and outcomes in lower extremity injury: A literature and systematic review. Journal of Athletic Training 47(3), 339-357.

8. Lynn S.K., Padilla R.A., Tsang K.W. (2012). Differences in static- and dynamic-balance task performance after 4 weeks of intrinsic-foot-muscle training: The short-foot exercise versus the towel-curl exercise. Journal of Sport Rehabilitation 21(4), 327-333.

9. Hong C., Guo L., Song R., Nagurka M.L., Sung J., Yen C. (2016). Assessing postural stability via the correlation patterns of vertical ground reaction force components. Biomedical Engineering Online 15(1), 90. DOI: 10.1186/s12938016-0212-z.

10. Romero-Franco N., Martínez-López E.J., Hita-Contreras F., Lomas-Vega R., Martínez-Amat A. (2015). Effects of an anaerobic lactic training session on the postural stability of athletes. The Journal of Sports Medicine and Physical Fitness 55(6), 578-586.

11. Sgrò F., Licari D., Coppola R., Lipoma M. (2015). Assessment of balance abilities in elderly people by means of a clinical test and a low-cost force plate. Kinesiology 47(1), 33-43.

12. Sousa A.P., Silva A., Tavares J.S. (2012). Biomechanical and neurophysiological mechanisms related to postural control and efficiency of movement: A review. Somatosensory $\mathcal{E}$ Motor Research 29(4), 131-143.

13. Cachupe W.C., Shifflett B., Kahanov L., Wughalter E.H. (2001). Reliability of Biodex Balance System measures. Measurement in Physical Education E Exercise Science 5(2), 97-108.

14. Cuğ M., Özdemir R.A., Ak E. (2014). Influence of leg dominance on single-leg stance performance during dynamic conditions: An investigation into the validity of symmetry hypothesis for dynamic postural control in healthy individuals. Turkish Journal of Physical Medicine E Rehabilitation 60(1), 22-26.
15. Didier J.J., Glave A.P., Browning S.J., Fiaud V., Weatherwax J. (2014). Reliability of BBS LOS test at two time points in a healthy population. Journal of Fitness Research 3(3), 3-7.

16. Ibrahim M.S., Mattar A.G., Elhafez S.M. (2016). Efficacy of virtual reality-based balance training versus the Biodex Balance System training on the body balance of adults. Journal of Physical Therapy Science 28(1), 20-26.

17. Danielsson T. (2010). Asymmetry in elite snowboarders. A study comparing range of motion in the hip and spine, power in lower extremities and circumference of thigh. Bachelor's thesis, Halmstad University School of Business and Engineering, Halmstad.

18. Staniszewski M., Zybko P., Wiszomirska I. (2016). Evaluation of laterality in the snowboard basic position. Human Movement 17(2), 119-125.

19. LeMaster R. (2010). Ultimate skiing. Champaign, IL: Human Kinetics.

20. Hrysomallis C. (2011). Balance ability and athletic performance. Sports Medicine 41(3), 221-232.

21. Platzer H., Raschner Ch., Patterson C., Lembert S. (2009). Comparison of physical characteristics and performance among elite snowboarders. Journal of Strength and Conditioning Research 23(5), 1427-1432.

22. Anthony C.C., Brown L.E., Coburn J.W., Galpin A.J., Tran T.T., Meir R. et al. (2016). Stance affects balance in surfers. International Journal of Sports Science \& Coaching 11(3), 446-450.

23. Čech P. (2014). Effect of short-term balance training on postural stability in ice hockey players. Acta Universitatis Carolinae: Kinanthropologica 50(2), 13-20.

24. Rajkumar P.S., Radhakrishnan T. (2016). Effect of proprioceptive training on static balance and dynamic balance of junior hockey players. International Journal of Science and Research 5(8), 936-939.

25. Karakaya M.G., Rutbİl H., Akpinar E., Yildirim A., Karakaya İ.Ç. (2015). Effect of ankle proprioceptive training on static body balance. Journal of Physical Therapy Science 27(10), 3299-3302.

26. Vando S., Filingeri D., Maurino L., Chaabène H., Bianco A., Salernitano G. et al. (2013). Postural adaptations in preadolescent karate athletes due to a one week karate training camp. Journal of Human Kinetics 38, 45-52.

27. Matin B.K., Yalfani A., Gandomi F., Abbasi H., Parmoon A. (2014). Neuromuscular training as the basis for developing the level of the static and dynamic balance in selected students of physical fitness team of Kermanshah. International Journal of Sports Sciences \& Fitness 4(1), 20-38.

28. Williams V.J., Nagai T., Sell T.C., Abt J.P., Rowe R.S., McGrail M.A. et al. (2016). Prediction of dynamic postural stability during single-leg jump landings by ankle and knee flexibility and strength. Journal of Sport Rehabilitation 25(3), 266-272.

29. Zemková E. (2014). Sport-specific balance. Sports Medicine 44(5), 579-590.

30. Williams D.S.B., Murray N.G., Powell D.W. (2016). Athletes who train on unstable compared to stable surfaces exhibit unique postural control strategies in response to balance perturbations. Journal of Sport and Health Science 5, 70-76.

Submitted: March 6, 2017

Accepted: April 26, 2017 